\title{
Cardiology
}

\section{Changes in Indicators of Oxidative Stress, Inflammation and Endothelial Dysfunction in Patients with Coronary Heart Disease during Lipid-Lowering Therapy}

\author{
Julia A. Kotova, PhD*; Anna A. Zuikova, PhD, ScD; Aleksander N. Pashkov, PhD, ScD \\ Voronezh State Medical University named after N.N. Burdenko \\ Voronezh, Russian Federation
}

\begin{abstract}
Results of this study present the features of the severity of coronary atherosclerosis, evaluated by the Gensini score, depending on the use of statins. Differences in indicators of oxidative stress, inflammation and endothelial dysfunction are revealed. In addition, the study established the relationship between statin intake and studied biomarkers and revealed the features of changes in the studied markers, depending on the achievement of the target levels of total cholesterol and LDL-C. (International Journal of Biomedicine. 2019;9(3):202-204.)
\end{abstract}

Key Words: coronary heart disease $\bullet$ oxidative stress $\bullet$ inflammation $\bullet$ endothelial dysfunction $\bullet$ lipid-lowering therapy

\section{Abbreviations}

CHD, coronary heart disease; DNPH, 2.4-dinitrophenylhydrazine; ED, endothelial dysfunction; GS, Gensini score; HDL-C, high-density lipoprotein cholesterol; hsCRP, high-sensitivity C-reactive protein; KDPH, ketone derivative of DNPH; LLT; lipid-lowering therapy; LDL-C, low-density lipoprotein cholesterol; OS, oxidative stress; TC, total cholesterol; RCVR, residual cardiovascular risk; SOD, superoxide dismutase; TC, total cholesterol; TG, triglycerides; tHcy, total homocysteine.

\section{Introduction}

Atherosclerosis, which is the primary pathophysiologic mechanism for the development of coronary heart disease (CHD), is a multifactorial and multifaceted process. (1-4) $^{(-1)}$ Currently, statins are the basis of primary and secondary prevention of coronary atherosclerosis. In addition to the fact that statins reduce the levels of total cholesterol (TC) and LDL-C and promote regression of atherosclerotic plaques, the use of statins is associated with proven efficacy against major cardiovascular events. However, the development of cardiovascular complications on the background of lipid-

*Corresponding author: Julia A. Kotova, PhD. Voronezh State Medical University named after N.N. Burdenko. Voronezh, the Russian Federation.E-mail: kotova_u@inbox.ru lowering therapy (LLT) with the achievement of target levels for LDL-C can be considered in the framework of the residual risk. ${ }^{(5)}$

Residual cardiovascular risk (RCVR) is defined as the risk of cardiovascular events that is present in patients, regardless of the achievement of the target levels for LDL-C, glucose and blood pressure on the background of standard therapy ${ }^{(6-8)}$ Even against the backdrop of intensive LLT, 70\% of patients still have a high RCVR..$^{(9,10)}$ In this regard, at the present stage, it is of interest to search for markers and combine them into risk determination algorithms to create new scales for cardiovascular risk stratification with the subsequent development of preventive measures. ${ }^{(11)}$

The aim of the study was to evaluate the changes in clinical and biochemical parameters in patients with different severity of coronary atherosclerosis, depending on the administration of LLT. 


\section{Materials and Methods}

We examined 354 patients (175 women and 179 men aged between 47 and 75 years, mean age of $61.8 \pm 8.1$ years) with CHD verified by standardized validated criteria and clinical-functional methods.

All patients underwent the following examinations: assessment of traditional risk factors, physical examination, clinical and biochemical laboratory methods, 12-lead ECG, echocardiography, Holter ECG monitoring, treadmill test, and coronary angiography. Blood samples were obtained in the morning after a $12 \mathrm{~h}$ overnight fast. The levels of total cholesterol (TC), low-density lipoprotein cholesterol (LDL-C), high-density lipoprotein cholesterol (HDL-C), triglycerides (TG) were determined in the blood plasma using "Daytona» analyzer (RANDOX, Ireland). CAG was performed by the Judkins technique using General Electric Innova 3100 (GE Healthcare, USA).

The severity of coronary atherosclerosis was evaluated by the Gensini score (GS). ${ }^{(12)}$ The severity of the disease is expressed as the sum of the scores for individual lesions and the functional importance index of the area of each lesion in the coronary tree. The sample was continuous.

According to the calculated GS, patients were divided into three groups: GS0 - 152 patients with normal coronary artery, GS1 (1-15 points) - 124 patients with hemodynamically insignificant coronary atherosclerosis, and GS2 ( $>15$ points) - 78 patients with hemodynamically significant coronary atherosclerosis.

Depending on the use of LLT (atorvastatin $40 \mathrm{mg}$ or rosuvastatin $20 \mathrm{mg}$ or simvastatin $40 \mathrm{mg}$ ), the patients were divided into 2 groups: Group 1 included 185 patients who did not receive statins for 6 months before the study; Group 2 included 169 patients who received statins for 6 months before the study.

The determination of oxidative modified proteins in the blood serum was carried out using the methods by Dubinina et al. ${ }^{(13)}$ The assay is based on the spectrophotometric detection of the reaction between 2.4-dinitrophenylhydrazine (DNPH) with protein carbonyl to form protein hydrazone. The optical density of 2,4-dinitrophenylhydrazones derivates was recorded on an SF-36 spectrophotometer. The optical density of aldehyde- and ketone derivatives of a neutral character was recorded at $356 \mathrm{~nm}$ and $370 \mathrm{~nm}$, respectively (ADPHn and KDPHn). The optical density of aldehyde- and ketone derivatives of a basic character was recorded at $430 \mathrm{~nm}$ and $530 \mathrm{~nm}$, respectively (ADPHb and $\mathrm{KDPHb}$ ).

The SOD activity was determined by the spectrophotometric method. The serum level of tHcy was determined by EIA using «Axis-Shield» test kit. The level of high-sensitivity C-reactive protein (chCRP) was determined using the High Sensitive Elisa Kit for CRP (Cloud-Clone Corp., USA).

Statistical analysis was performed using statistical software package SPSS version 20.0 (SPSS Inc, Chicago, IL). Median values are presented with interquartile (IQ) ranges (IQR; 25th to 75th percentiles). The Mann-Whitney test was used to compare median values. The frequencies of categorical variables were compared using Pearson $\chi^{2}$. The Spearman correlation coefficient $\left(\mathrm{r}_{\mathrm{s}}\right)$ was used to assess the relationship between variables. A probability value of $P<0.05$ was considered statistically significant.

\section{Results and Discussion}

All patient groups were matched for age. In Group 1, GS0 was determined in 88 patients, GS0-GS1 in 34 patients and GS2 in 47 patients. In Group 2, GS0 was determined in 64 patients, GS0-GS1 in 90 patients, and GS2 in 31 patients (Fig.1). A statistical relationship was found between a group of patients separated by GS and statin intake $\left(\chi^{2}[\mathrm{df}=2]=13.432\right.$, $\phi=0.283, P=0.0012$ ) There were 2.6 times more patients with GS0 and GS1 who took statins compared to patients without statins. The reverse trend was found in the GS2 group: there were 1.8 times more patients who did not take statins than those who did. In the GS0 group, in $58 \%$ of cases patients did not take statins.

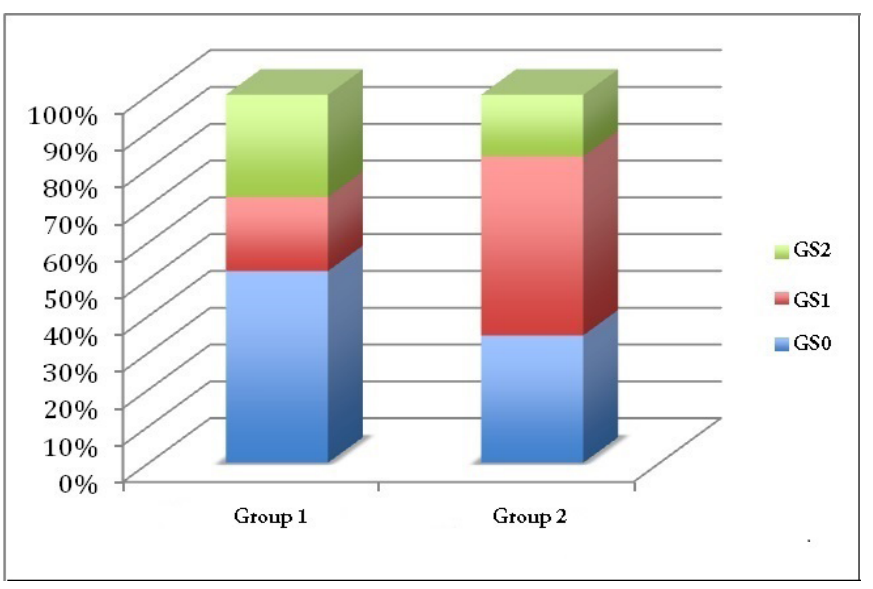

Fig.1. The structure of the studied groups depending on the severity of coronary atherosclerosis.

Among patients treated with statins, the average score, which determined the level of GS, was 10.5[8;23.6], and among patients who did not take statins - 26[14;31] points. When evaluating clinical and anthropometric indices, significant differences were established in BMI $(P=0.000)$, waist circumference $(P=0.000)$, and systolic blood pressure $(P=0.25)$. When assessing the lipid profile, significant differences were found in the levels of TC $(P=0.001)$ and HDL-C $(P=0.021)$ between the studied groups. There were no significant differences in the levels of TG and LDL-C.

Comparison of parameters of OS, hsCRP and tHcy in patients with signs of coronary lesion and statin intake or no intake ( $\mathrm{St}+$ and $\mathrm{St}-$ ) is presented in Table 1. A significant difference was found for all studied parameters. There was no such tendency among patients without signs of coronary atherosclerosis: significant differences were found only for the levels of tHcy and SOD.

Correlation analysis in patients with signs of CA revealed significant correlations between statin intake and levels of tHcy $\left(\mathrm{r}_{\mathrm{s}}=-0.502, P=0.000\right)$, hsCRP $\left(\mathrm{r}_{\mathrm{s}}=-0.329, P=0.002\right)$, SOD 
$\left(\mathrm{r}_{\mathrm{s}}=0.228, P=0.033\right)$, ADPHn $\left(\mathrm{r}_{\mathrm{s}}=-0.307, P=0.004\right)$, KDPHn $\left(\mathrm{r}_{\mathrm{s}}=-0.243, P=0.023\right)$, ADPHb $\left(\mathrm{r}_{\mathrm{s}}=-0.394, P=0.000\right), \mathrm{KDPHb}$ $\left(\mathrm{r}_{\mathrm{s}}=-0.237, P=0.027\right)$, TC $\left(\mathrm{r}_{\mathrm{s}}=-0.344, P=0.001\right)$, and HDL-C $\left(\mathrm{r}_{\mathrm{s}}=0.249, P=0.02\right)$. In patients without signs of coronary atherosclerosis, significant correlations were found between statin intake and levels of tHcy $\left(\mathrm{r}_{\mathrm{s}}=-0.361, P=0.001\right)$ and SOD $\left(\mathrm{r}_{\mathrm{s}}=0.286, P=0.010\right)$.

\section{Table 1.}

The level of biochemical markers among patients with coronary atherosclerosis, depending on the use of statins

\begin{tabular}{|l|c|c|c|}
\hline \multicolumn{1}{|c|}{ Variable } & St + & St- & $\mathrm{P}$ \\
\hline tHcy, $\mu \mathrm{mol} / \mathrm{l}$ & $11.34[10.36 ; 11.98]$ & $11.80[10.84 ; 12.37]$ & 0.000 \\
\hline hsCRP, $\mathrm{mg} / 1$ & $0.21[0.12 ; 0.76]$ & $0.43[0.03 ; 0.85]$ & 0.002 \\
\hline SOD, $\%$ & $35.21[32.41 ; 37.30]$ & $34.89[32.05 ; 37.04]$ & 0.034 \\
\hline ADPHn, IU/mg & $25.37[24.21 ; 27.31]$ & $26.74[24.21 ; 28.01]$ & 0.004 \\
\hline KDPHn, IU/mg & $21.43[20.32 ; 22.74]$ & $22.74[20.65 ; 23.98]$ & 0.024 \\
\hline ADPHb, IU/mg & $11.03[10.72 ; 11.75]$ & $11.43[10.98 ; 12.34]$ & 0.000 \\
\hline KDPHb, IU/mg & $6.98[6.34 ; 8.76]$ & $7.32[6.72 ; 8.76]$ & 0.028 \\
\hline
\end{tabular}

Analysis of the achievement of the target levels of lipid blood spectrum in patients with signs of coronary atherosclerosis revealed a statistically significant relationship between the achievement of the target levels of TC and LDP-C and statin intake $\left(\chi^{2}[\mathrm{df}=2]=5.649, \phi=0.255, P=0.017\right.$; $\chi^{2}[\mathrm{df}=2]=7.853, \phi=0.300, P=0.005$; respectively). Among patients who took statins, TC $<5 \mathrm{mmol} / \mathrm{l}$ was reached in $69.6 \%$ of cases and LDL-C $<1.8 \mathrm{mmol} / 1$ in $82.6 \%$ of cases. When comparing the studied markers, depending on the achievement of the target level for TC, significant differences were found for all parameters $(P<0.05)$. With the achievement of the target level for LDL-C, the levels of tHcy $(P=0.006)$ and KDPHn $(P=0.05)$, an early marker of OS, significantly changed.

\section{Conclusion}

Thus, in clinical practice, the therapy of the atherogenic dyslipoproteinemias remains at a suboptimal level, and a significant proportion of patients do not reach the target levels of TC and LDL-C. Low adherence to LLT in patients with hemodynamically significant coronary atherosclerosis was noted. In this regard, the search for RCVR markers, taking into account the significance of non-lipid risk factors, remains necessary.

\section{Competing Interests}

The authors declare that they have no competing interests.

\section{Sources of Funding}

This work was partially supported by the Council on Grants of the President of the Russian Federation for State Support of Young Scientists and Leading Scientific Schools (Grant MK-552.2018.7).

\section{References}

1. Ragino YuI, Chernyavskij AM, Eremenko NV, Shakhtshnejder EV, Polonskaya YaV, Tsimbal SYu, et al. [Key laboratory diagnostic biomarkers of coronary atherosclerosis]. Kardiologiia. 2011;51(3): 42-6. [Article in Russian].

2. Vertkin AL, Topolyanskij AV. [The problem of hyperhomocysteinemia in cardiac patients]. Farmateka. 2007;15:10-14. [Article in Russian].

3. L'vovskaya EI, Sahankova EN. [The ratio of levels of lipid peroxidation and oxidative modification of proteins in students 17-23 years (Kungur)]. Vestnik YUrGU. 2012; 21: 112-116. [Article in Russian].

4. Musthafa QA, Abdul Shukor MF, Ismail NAS, Mohd Ghazi A, Mohd Ali R, M Nor IF, et al. Oxidative status and reduced glutathione levels in premature coronary artery disease and coronary artery disease. Free Radic Res. 2017;51(9-10):787798. doi: 10.1080/10715762.2017.1379602.

5. Tian J, Gu X, Sun Y, Ban X, Xiao Y, Hu S, Yu B. Effect of statin therapy on the progression of coronary atherosclerosis. BMC Cardiovasc Disord. 2012;12:70.

6. Diagnostics and correction of lipid metabolism disorders in order to prevent and treat atherosclerosisю Russian recommendations VI revision. The Journal of Atherosclerosis and Dyslipidemias.2017;3:5-23.[in Russian].

7. Blankstein R, Budoff MJ, Shaw LJ, Goff DC Jr, Polak JF, Lima J, et al. Predictors of coronary heart disease events among asymptomatic persons with low low-density lipoprotein cholesterol MESA (Multi-Ethnic Study of Atherosclerosis). J Am Coll Cardiol. 2011;58(4):364-74. doi: 10.1016/j. jacc.2011.01.055.8.

8. Kones R. Molecular sources of residual cardiovascular risk, clinical signals, and innovative solutions: relationship with subclinical disease, undertreatment, and poor adherence: implications of new evidence upon optimizing cardiovascular patient outcomes. Vasc Health Risk Manag. 2013;9:617-70. doi: 10.2147/VHRM.S37119.

9. Fruchart JC, Davignon J, Hermans MP, Al-Rubeaan K, Amarenco P, Assmann G; Residual Risk Reduction Initiative (R3i). Residual macrovascular risk in 2013: what have we learned? Cardiovasc Diabetol. 2014;13:26. doi: 10.1186/14752840-13-26.10.

10. National Cholesterol Education Program (NCEP) Expert Panel on Detection, Evaluation, and Treatment of High Blood Cholesterol in Adults (Adult Treatment Panel III). Third Report of the National Cholesterol Education Program (NCEP) Expert Panel on Detection, Evaluation, and Treatment of High Blood Cholesterol in Adults (Adult Treatment Panel III) final report. Circulation. 2002;106(25):3143-421.

11. Dallmeier D, Koenig W. Strategies for vascular disease prevention: the role of lipids and related markers including apolipoproteins, low-density lipoproteins (LDL)particle size, high sensitivity C-reactive protein (hs-CRP), lipoprotein-associated phospholipase A2 (Lp-PLA ${ }_{2}$ ) and lipoprotein(a) (Lp(a)). Best Pract Res Clin Endocrinol Metab. 2014;28(3):281-94. doi: 10.1016/j.beem.2014.01.003.

12. Gensini GG. A more meaningful scoring system for determining the severity of coronary heart disease. Am J Cardiol. 1983;51(3):606.

13. Dubinina EE, Burmistrov SO, Khodov DA, Porotov IG. [Oxidative modification of human serum proteins. A method of determining it].Vopr Med Khim. 1995;41(1):24-6. [Article in Russian]. 